\title{
Informationen aus der DMV-Geschäftsstelle
}

Liebe DMV-Mitglieder,

folgende Informationen aus der Geschäftsstelle möchten wir Ihnen gerne mitteilen.

\section{Aktualisierung Ihrer Adressdaten}

Bitte kontrollieren Sie Ihre persönlichen Angaben auf unserer Homepage. Sie können Ihre persönlichen Daten jederzeit selbstständig ändern, wenn Sie eingeloggt sind. Änderungen können auch per Mail oder Formular (www.mathematik. de/mitgliedsdaten-aendern) mitgeteilt werden.

Mitteilung über die Beitragseinziehung 2020

DMV: Januar/Februar 2020

EMS: $\quad$ Februar 2020

FG Stochastik: Februar 2020

DMV-Mitglieder mit studentischem Mitgliedsbeitrag

Bitte beachten Sie, dass Sie für die Inanspruchnahme des reduzierten Mitgliedsbeitrags einen Nachweis vorlegen müssen.

Bitte an alle Einzahler

Einzahlungen müssen extra gebucht und ggf. gemahnt werden; das entfällt, wenn Sie uns eine Einzugsermächtigung erteilen. Das Formular für das SEPA-Lastschriftmandat finden Sie auf der DMV-Homepage unter www.mathematik. de/mitgliedsdaten-aendern.

Einzahlungen bitte nur auf das Konto:

Volksbank Freiburg, IBAN DE66 6809 oooo ooo6 9550 02, BIC GENODE61FR1.

Beitragszahlung für DMV-Mitglieder aus Übersee Nur für DMV-Mitglieder aus Übersee besteht die Möglichkeit, Ihren Mitgliedsbeitrag per PayPal zu entrichten, falls keine Kreditkarte zur Verfügung steht. Dazu müssen Sie eingeloggt sein: www.mathematik.de/ mitgliedsbeitrag-bezahlen
Bemerkung zur steuerlichen Behandlung der Mitgliedsbeiträge Für den Nachweis beim Finanzamt reicht in der Regel Ihr Kontoauszug. Mitgliedsbeitrag im Sinne von §1ob Abs. 1 EStG (Abzug als Sonderausgaben) ist der Mitgliedsbeitrag abzgl. der Kosten für die gewählte(n) Zeitschrift(en) und der DMV-Mitteilungen. Die DMV-Mitteilungen werden mit $10 €$ incl. $7 \%$ Umsatzsteuer angesetzt. Daneben kommt der Abzug des gesamten Mitgliedsbeitrags einschließlich der Kosten für die Zeitschriften als Werbungskosten in Betracht.

Werbung neuer DMV-Mitglieder

Wir möchten alle DMV-Mitglieder aufrufen, in ihrem Umfeld für die DMV zu werben.

DMV-Flyer, DMV-Gutscheine für eine kostenlose Ausgabe der DMV-Mitteilungen sowie Informationsmaterial über die DMV und einen DMV-Foliensatz über die Aktivitäten der DMV zum Herunterladen finden Sie unter www.mathematik.de/dmv-infopaket.

\section{Spenden an die DMV}

Damit die vielen Projekte der DMV (z. B. Nachwuchsarbeit, Preise- und Auszeichnungen etc.) verwirklicht werden können, ist die DMV auf Spenden angewiesen! Unter folgendem Link können Sie die Spenden einzahlen: www.mathematik.de/spenden

Bescheinigungen über den von Ihnen gezahlten Spendenbetrag stellen wir Ihnen gerne aus.

Wir bedanken uns für Ihre Mitgliedschaft! 